\title{
Label-free visualization of heterogeneities and defects in metal-organic frameworks using nonlinear optics ${ }^{\dagger}$
}

Received 00th January 20xx, Accepted 00th January 20xx DOI: $10.1039 / \times 0 \times x 00000 x$

\author{
Mathias Wolf, a Kenji Hirai, ${ }^{* b}$ Shuichi Toyouchi, ${ }^{\text {a }}$ Eduard Fron, ${ }^{a}$ Wannes Peeters, ${ }^{a}$ \\ Steven De Feyter ${ }^{a}$ and Hiroshi Uji-i*a,b
}

Defects influence the properties of metal-organic frameworks (MOFs) e.g. storage amount and diffusion kinetics of gas molecules. However, the spatial distribution of defects is still poorly understood due to a lack of visualization methods. Here, we present a new method using nonlinear optics (NLO) allowing visualization of defects within MOFs.

Metal-organic frameworks (MOFs) are a type of porous crystalline materials, consisting of metal nodes connected by organic linkers. MOFs are used for applications such as gas storage and separation as well as catalysis due to their high surface area. ${ }^{1-5}$ However, their flexible structures also make them prone to develop defects during synthesis or after removal from a solvent and exposure to air. ${ }^{6,7}$ These defects have a large impact on the performance of MOFs and affect their intended purposes. ${ }^{8,9}$ However, their spatial distribution is still not well understood. It is likely that the position of defects (e.g. at the edge vs. the centre of a crystal) has a large influence on the alteration of properties and thus methods for visualizing defects on a scale ranging from the nanometer to micrometer are necessary to fully understand the impact of defects.

Scanning electron microscopy (SEM) can give spatia information about inhomogeneities with great resolution but penetration depth is limited and it requires high vacuum which can cause additional defects. ${ }^{10}$ Ameloot et al. have shown that confocal fluorescence microscopy can be used to obtain a 3D image of MOF heterogeneities and defects. ${ }^{11}$ Moreover, this method revealed that heterogeneities and defects exist in a crystal, even though it appears as a perfect single crystal under

\footnotetext{
a. Department of Chemistry, KU Leuven, Celestijnenlaan 200F, 3001 Heverlee, Belgium.E-mail: hiroshi.ujii@kuleuven.be

b. Research Institute for Electronic Science (RIES), Hokkaido University, N20W10,

Kita ward, Sapporo, 001-0020 Hokkaido, Japan Address here. E-mail: hirai@es.hokudai.ac.jp

†Electronic Supplementary Information (ESI) available: [details of any supplementary information available should be included here]. See DOI: $10.1039 / \times 0 \times x 00000 x$
}

optical transmission microscope. ${ }^{11}$ In spite its simplicity, this method still requires the introduction of fluorescent probe molecules. Depending on the chemical nature of the defect, such labelling may not always be easily possible, the pores of MOFs could be too small for fluorescent molecules to diffuse inside the crystal or the MOF itself could be fluorescent overshadowing fluorescence signal from probe molecules. Therefore, there is a need for non-destructive label-free methods that provide information on spatial inhomogeneities and defects in these materials.

Imaging techniques based on nonlinear optical (NLO) phenomena might be well-suited. Nonlinear optics refers to a field in which a material responds in a nonlinear way to an electric field of light. This nonlinearity means that the material response typically has higher order contributions. These higher orders give rise to a variety of effects, e.g. second- and thirdharmonic generation (SHG and THG, respectively), sumfrequency generation (SFG) and four-wave mixing (FWM). It is well-known that $2^{\text {nd }}$ order effects like SHG and SFG only occur in non-centrosymmetric materials or at interfaces, while $3^{\text {rd }}$ order effects, such as THG and FWM, can appear for all structures. Furthermore, under tight-focusing conditions THG and FWM display different phase matching; THG highlights the spatial variations in the third-order nonlinear susceptibility $\left(\Delta \chi^{(3)}\right)$ that is often enhanced at interfaces, while FWM directly detects the spatial distribution of $\chi^{(3)} \cdot{ }^{12-14}$ Mahou et al. have demonstrated these different phase-matching conditions by imaging unstained tissue of Caenorhabditis elegans worms. ${ }^{14}$ So as NLO for visualization does not require any labelling, can give multiple information with just one measurement, has greater resolution and penetration depth compared to conventional fluorescence microscopy and unlike SEM does not require vacuum conditions. ${ }^{14-17}$ it might be well-suited for the investigation of MOF heterogeneities and defects.

In this study, we demonstrate the use of NLO to visualize different types of defects and heterogeneities on the scale of 
1 $\gamma$-CD MOF

a

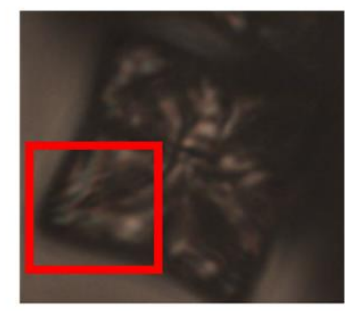

b

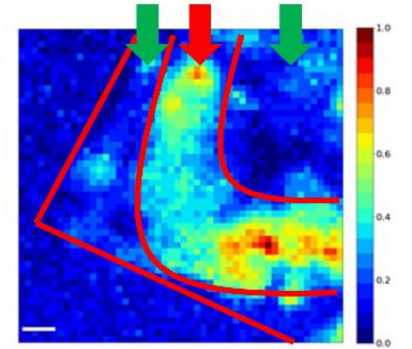

2

MOF-177

C
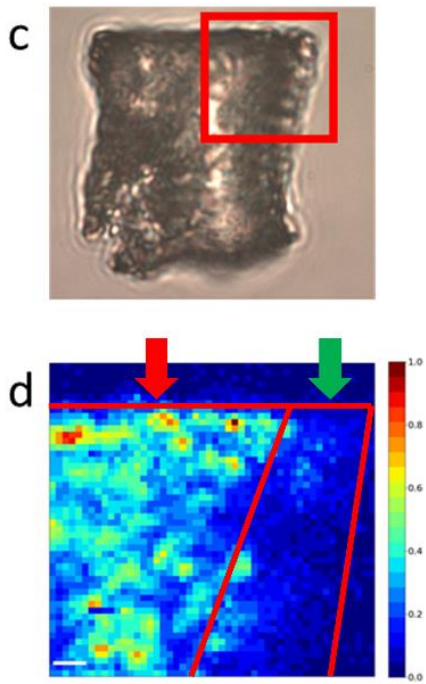

3

CoTPT

e

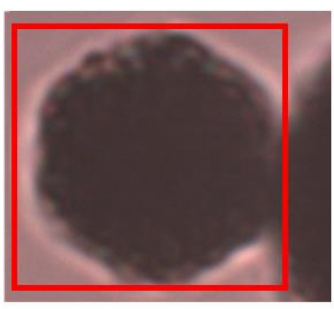

f

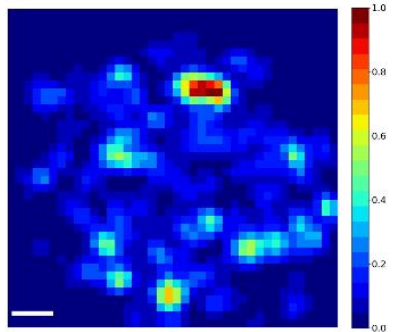

Figure 1 a), c), e) Optical transmission images of $\mathbf{1}, \mathbf{2}$ and $\mathbf{3}$ respectively. b), d), f) Maps showing the intensity distribution of FWM of $\mathbf{1}, \mathbf{2}$ and $\mathbf{3}$ respectively. Maps were obtained from the area marked by the red square in the corresponding transmission images. Scalebars in $b, d$ and $f$ are 1 $\mu \mathrm{m}$. Red and green arrows in $\mathrm{b}$ and $\mathrm{d}$ point to bright and dark regions respectively. ${ }^{\ddagger}$

hundreds of nanometers to micrometers in MOFs and how indepth information about MOFs' structure and composition can be obtained. We also show the influence of guest accommodation on MOF heterogeneities.

We synthesized microcrystals of CD-MOF (1), MOF-177 (2) and CoTPT (3). Optical transmission images of $\mathbf{1}, \mathbf{2}$, and $\mathbf{3}$ are shown in figs. 1a, 1c, and 1e (see figs. S2-4 for molecular structures, figs. S5-8 for characterization by SEM and XRD).

When irradiating a sample with two pulsed lasers with different wavelengths, it is typically possible to observe several NLO signals at once. In the case of this measurement, the sample is irradiated by femtosecond pulses at $820 \mathrm{~nm}$ and $1164 \mathrm{~nm}$. Depending on the samples and the relative difference in intensity between the two beams, signals can be detected at $632 \mathrm{~nm}$ (FWM), $582 \mathrm{~nm}$ (SHG), $480 \mathrm{~nm}$ (SFG), $410 \mathrm{~nm}$ (SHG) and $388 \mathrm{~nm}$ (THG). If the sample is fluorescent and has a considerable two-photon absorption cross-section it is also possible to observe two-photon luminescence. Among the various NLO signals, FWM is a suitable technique for visualization because it can be obtained from any sample without symmetry restrictions. Measuring FWM gives a map of the distribution of the third-order nonlinear susceptibility $\chi^{(3)} \cdot \chi^{(3)}$ is a $4^{\text {th }}$ rank tensor with 81 elements that is usually non-zero for all materials. The exact strength of FWM typically depends on the polarization and the propagation direction with respect to the crystal axes. However, these are only important if efficient wave-mixing, meaning maximum conversion from fundamental to produced beam, is desired. Typically, using phase-matching techniques allows NLO signal generation over large domains. Phase-matching techniques are not needed in the case of NLO microscopy as the interaction volume is limited to the focus region of the beam which is usually in the range of the coherence length without any phase-matching. ${ }^{12}$ Therefore, perfectly crystalline material will display spatially homogeneous FWM signal independent of phase-matching conditions and thus signal changes can be associated with inhomogeneities.

Figs. 1b, $d$ and $f$ display FWM maps obtained from 1, 2 and 3, respectively. The corresponding mapping areas are indicated with the red squares in the transmission images (figs. 1a, 1c, and 1e). The different characteristics on FWM displayed by the three MOFs are very clear. 1 shows primarily three different regions, two "darker" ones at the edge and towards the centre as well as a brighter region between the two (fig. 1b, bright and dark regions are marked by red and green arrows respectively). This corresponds well with the transmission image in fig. 1a, with a darker edge and central regions and a transmissive part in between. While 1 shows more connected domains, $\mathbf{3}$ displays a foam-like structure (fig. 1f). The comparison with the transmission image (fig. 1e) is slightly more difficult here because the crystal is too thick for optical transmission imaging, however around the edges the foam-like characteristic of the MOF can be recovered in the transmission image as well.

The difference in FWM appearance and thus domain size is likely due to the number of nucleation points during the crystal formation.

As seen in fig. $1 \mathrm{~d}, \mathbf{2}$ has two more connected domains like 1, a bright one to the left and a dark one to the right (marked with red and green arrows in fig. $1 \mathrm{~d}$, respectively), but at the same time the bright domain also displays the foam-like image similar to 3. Comparing this with the transmission image (fig. 1c) it is now possible to see the connection between the transmission and FWM images. The bright region in FWM resembles the transmission part while the dark FWM region corresponds to the dark region in the transmission image. Similar to the 
a

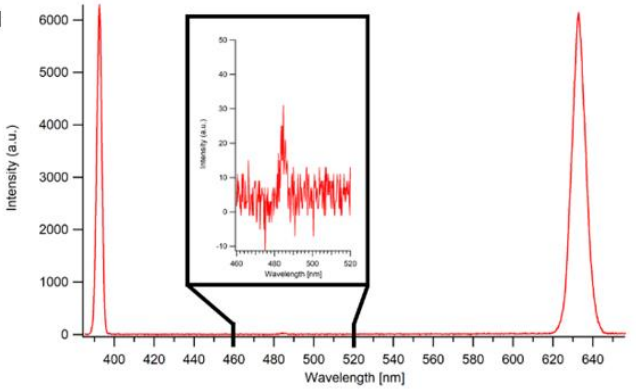

b
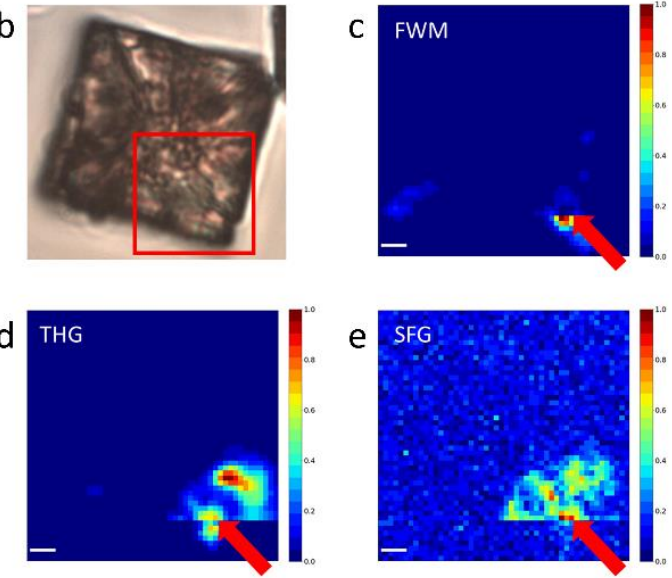

Figure 2 a) Example spectrum obtained during this measurement. The three peaks observed are at $632 \mathrm{~nm}$ (FWM), $480 \mathrm{~nm}$ (SFG) and $388 \mathrm{~nm}$ (THG). Excitation by $820 \mathrm{~nm}$ and $1164 \mathrm{~nm}$ fs pulses. b) Optical transmission image of $\left.\left.\left.1 \supset \mathrm{C}_{60} \mathrm{c}\right), \mathrm{d}\right), \mathrm{e}\right)$ Maps showing the intensity distribution of FWM, THG and SFG respectively. Maps were obtained from the area marked by the red square in $b$. Scalebars in c, $d$ and e are $1 \mu \mathrm{m}$.

transmission image, the foam-like nature can also be observed These results demonstrate the powerful nature of FWM method for imaging the inhomogeneities of MOFs. The technique can be used for a variety of MOFs and it works for thick samples that cannot be characterized with optical transmission images, fluorescence or SEM. The domain size can be estimated from the FWM map without requiring vacuum. One down-side of FWM is that it is not giving information about the specific nature of the inhomogeneity, because FWM simply reflects the distribution of third-order nonlinear susceptibility. By comparing different NLO signals, however, additional information can be extracted. An example of a spectrum with multiple NLO signals can be seen in fig. 2a obtained from 1 accommodating $\mathrm{C}_{60}\left(1 \supset \mathrm{C}_{60}\right)$, where FWM, SFG and THG are visible. Fig. 2 shows the transmission image of $1 \supset C_{60}(b)$ as well as the maps of FWM (c), THG (d) and SFG (e). All NLO signals have been obtained at the same time. All three NLO maps display a sharp edge in the lower right corner. Looking at the transmission image, it can be seen that there are two slightly twisted phases on top of each other. Because second-order effects only appear when centro-symmetry is broken, they are commonly used to image defects. Fig. 2e shows clear signal from the second-order SFG close to the aforementioned edge, indicating that there is a large number of defects in this region. The image shows a branch-like structure extending from a central point at the edge (marked by a red arrow in fig. 2e). To further understand the nature of this defect, FWM and THG maps can be used. The FWM image (fig. 2c) displays very different intensities below and above the edge, meaning that there must be a difference either in composition or structure. Looking at the THG map (fig. 2d), we can see high intensity above the edge. This points to significant spatial variations in the third-order nonlinear susceptibility $\left(\Delta \chi^{(3)}\right)$ in this region. Overall taking the findings together, we conclude from FWM that $\chi^{(3)}$ is very different in the top and bottom part of the crystal pointing to a stark difference in material composition, from SFG that there is a large number of defects in the upper part of the crystal, as well as from THG that within the top part of the crystal there are strong changes in the third-order nonlinear susceptibility $\Delta \chi^{(3)}$. It can be concluded that the features observed likely come from cracks in the crystal possibly due to stress induced by the morphing of two phases with different orientation angles.

As 1 belongs to a non-centrosymmetric space group, this demonstrates also the applicability of NLO microscopy and in particular second-order effects for non-centrosymmetric MOFs. Defects will typically present a larger breaking of centrosymmetry than an asymmetric crystal structure.

Finally, we investigated the effect of guest accommodation in MOFs by combining NLO with Raman scattering imaging. $\mathbf{3}$ has large pore apertures allowing it to accommodate $C_{60}$. $C_{60}$ is a molecule with strong Raman-scatter signal and its distribution can be probed by Raman mapping technique. ${ }^{18}$ Thus, 3 is an excellent candidate to demonstrate the effect of guest accommodation because NLO maps can be compared with $C_{60}$ Raman maps. It has been shown that $\mathbf{3}$ has a semi-amorphous structure under ambient conditions due to the evaporation of solvent molecules. ${ }^{19}$ When the pores are occupied by guest molecules, the framework structure of $\mathbf{3}$ could be stabilized. Indeed, XRD spectra of $\mathbf{3}$ with and without $\mathrm{C}_{60}$ as guest molecules display this stabilization effect by guest molecules. (see fig. $\mathrm{S} 8$ in ESI). Figs. 3a and b display example spectra obtained during measurement for $\mathrm{C}_{60}$-Raman and NLO signals respectively. For Raman mapping, the $\mathrm{C}_{60} \mathrm{~A}_{g}(2)$ mode peak at $1470 \mathrm{~cm}^{-1}$ is used. For NLO mapping, FWM at $632 \mathrm{~nm}$ and SHG at $582 \mathrm{~nm}$ are used. Both NLO signals were obtained at the same time but Raman spectra were taken separately using $785 \mathrm{~nm}$ CW excitation. Fig. 3c shows a transmission image. It should be noted that in the top part of the obtained maps (figs. 3d-f), two MOFs are slightly touching. It is necessary to look at these MOFs separately. Looking at the FWM map (fig. 3d) the foam-like structure similar to $\mathbf{3}$ (without guest molecule) could be observed again for $3 \supset C_{60}$. Comparing the spatial distribution of $\mathrm{C}_{60}$ Raman signal and SHG (figs. $3 e, f$ respectively), corresponding features can be observed. In the bottom MOF (white square in figs. $3 e, f$ ) the Raman signal is roughly homogeneous while only little SHG is observed pointing to an overall crystalline structure. But it should be noted that some SHG is still observed meaning defects are still present. The fact 
a
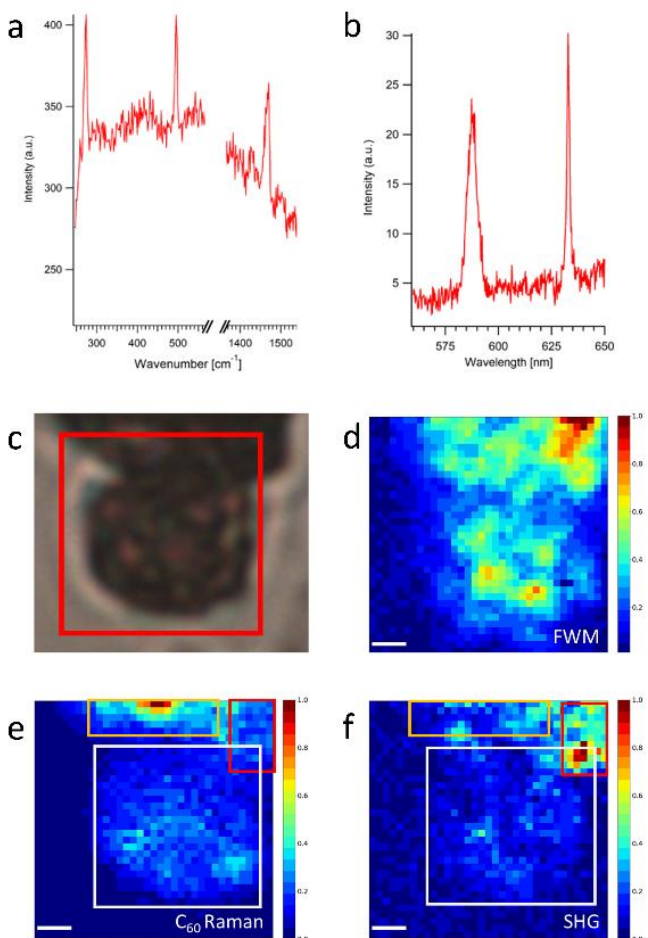

Figure 3 a) Example of a Raman spectrum obtained during this measurement. The three peaks shown originate from $\mathrm{C}_{60}: \mathrm{H}_{g}(1)$ at $274 \mathrm{~cm}^{-1}, \mathrm{~A}_{g}(1)$ at $495 \mathrm{~cm}^{-1}$ and $\mathrm{A}_{g}(2)$ at $1470 \mathrm{~cm}^{-1}$. For mapping, the $A_{g}(2)$ mode peak was used. b) Example of a NLO spectrum obtained during this measurement. The two peaks observed are at 632 $\mathrm{nm}$ (FWM) and $588 \mathrm{~nm}$ (SHG). c) Optical transmission image of $3 \mathrm{JC}_{60}$ d), e), f) Maps showing the intensity distribution of FWM, Raman $A_{g}(2)$ mode of $\mathrm{C}_{60}$ and SHG respectively. Maps were obtained from the area marked by the red square in a. Scalebars in $\mathrm{d}$, e and f are $1 \mu \mathrm{m}$. ${ }^{\ddagger}$

that defects remain even after guest accommodation could not be observed using XRD showing the higher power of NLO. In the top MOF we can see clear anti-correlation between Raman signal and SHG. Looking at the area marked in the orange square (figs. $3 e, f$ ), it shows high intensity in $\mathrm{C}_{60}$ Raman but only weak signal of SHG indicating high crystallinity. The red squares in the same images show the opposite, weak $C_{60}$ Raman signal but strong SHG. Stronger Raman signal indicates more $\mathrm{C}_{60}$ is present that in turn stabilizes the structure and reduces SHG and vice versa (low Raman $=>$ high SHG). This is direct proof of stabilization of $\mathbf{3}$ through the accommodation of guest molecules. It should be noted that the Raman signals in the red and white squares (fig. $3 e$ ) should not be compared as they are from different crystals with different average intensities because of their different sizes.

We have shown the power of NLO for visualization of inhomogeneities and defects in MOFs. By using FWM an overall picture of the spatial distribution of the inhomogeneities of MOFs can be visualized. Additional information about chemical composition and structural defects can be obtained by considering THG and SFG/SHG. All of these can be imaged simultaneously by a single scan and provide insight into the defects and their distribution within MOFs, which might be of relevance for optimizing synthesis protocols of MOFs.

\section{Conflicts of interest}

There are no conflicts to declare.

\section{Acknowledgements}

This research was supported by the Research Foundation Flanders (FWO) project (G081916N, GOD4519N) and the KU Leuven Research Fund (C14/19/079 and C14/15/053). M.W. acknowledges the SB-PhD fellowship (1S87918N). This research was partially supported by the JSPS Kakenhi (JP 18H0194809, JP17H03003, JP20K21165) and JSPS Core-to-Core Program, A. Advanced Research Networks.

\section{Notes and references}

¥ All maps shown are $x, y$-slices obtained roughly $2 \mu \mathrm{m}$ above the MOF-coverslip interface. See fig. S1 for a detailed description.

1 S. Kitagawa, R. Kitaura and S. Noro, Angew. Chem. Int. Ed., 2004, 43, 2334

2 H. Furukawa, K.E. Cordova, M. O'Keeffe and O.M. Yaghi, Science, 2013, 341

3 A. Schneemann, V. Bon, I. Schwedler, I. Senkovska, S. Kaskel and R.A. Fischer, Chem. Soc. Rev., 2014, 43, 6062

4 H. Li, M.M. Sadiq, K. Suzuki, R. Ricco, C. Doblin, A.J. Hill, S. Lim, P. Falcaro and M.R. Hill, Adv. Mater., 2016, 28, 1839

5 Y.B. He, W. Zhou, G.D. Qian and B.L. Chen, Chem. Soc. Rev., 2014, 43, 5657

6 W.H. Zhang, M. Kauer, P.H. Guo, S. Kunze, S. Cwik, M. Muhler, Y.M. Wang, K. Epp, G. Kieslich and R.A. Fischer, Eur. J. Inorg. Chem., 2017, 925

7 K. Müller, N. Vankova, L. Schöttner, T. Heine and L. Heinke, Chem. Sci., 2019, 10, 153

8 G.G. Chang, X.C. Ma, Y.X. Zhang, L.Y. Wang, G. Tian, J.W. Liu, J. Wu, Z.Y. Hu, X.Y. Yang and B.L. Chen, Adv. Mater., 2019, 31

9 Z. Fang, B. Bueken, D.E. De Vos and R.A. Fischer, Angew. Chem. Int. Ed., 2015, 54, 7234

10 J. Ren, M. Ledwaba, N.M. Musyoka, H.W. Langmi, M. Mathe, S. Liao and W. Pang, Coord. Chem. Rev., 2017, 349, 169

11 R. Ameloot, F. Vermoortele, J. Hofkens, F.C. De Schryver, D.E. De Vos and M.B.J. Roeffaers, Angew. Chem. Int. Ed., 2013, 52, 401

12 J.X. Cheng, A. Volkmer and X.S. Xie, J. Opt. Soc. Am. B, 2002, 19, 1363

13 J.X. Cheng and X.S. Xie, J. Opt. Soc. Am. B, 2002, 19, 1604

14 P. Mahou, N. Olivier, G. Labroille, L. Duloquin, J.M. Sintes, N. Peyriéras, R. Legouis, D. Débarre and E. Beaurepaire, Biomed. Opt. Express, 2011, 2, 2837

15 X. Chen, S. Grégoire, F. Formanek, J.B. Galey and H. Rigneault, J. Control. Release, 2014, 200, 78

16 N. Mazumder, N.K. Balla, G.Y. Zhuo, Y.V. Kistenev, R. Kumar, F.J. Kao, S. Brasselet, V.V. Nikolaev and N.A. Krivova, Front. Phys., 2019, 7

17 L. Tong and J.X. Cheng, Mater. Today, 2011, 6, 264

18 M.S. Dresselhaus, G. Dresselhaus and P.C. Eklund, J. Raman Spectrosc., 1996, 27, 351

19 G. Brunet, D.A. Safin, I. Korobkov, A. Cognigni and M. Murugesu, Cryst. Growth Des., 2016, 16, 4043 